\title{
Effects of artificial rewarming upon hypothermia-induced retrograde amnesia
}

\author{
WILLIAM C. WETSEL, DAVID C. RICCIO, and CHARLES F. HINDERLITER \\ Kent State University, Kent, Ohio 44242
}

\begin{abstract}
Three experiments were conducted to examine the relationship between recovery rate from deep body cooling and later memory loss. In each of these three investigations, a passive avoidance trial was followed immediately by hypothermia and recovery was manipulated by exposing rats to warm water. In Experiment I, rats returned to near normothermic levels by immersion in $36.4^{\circ} \mathrm{C}$ water for $20 \mathrm{~min}$ following the training-hypothermia treatment showed good retention, while rats allowed to recover under room temperature conditions showed the typical RA. Results from Experiment II indicated that extent of amnesia was inversely related to the duration of immersion in warm water following training-hypothermia treatment. In Experiment III, the prevention of RA by rewarming was shown to follow a time-dependent course. Initiation of rewarming 0,5 , or $10 \mathrm{~min}$ after hypothermia treatment eliminated RA, but the same rewarming given $30 \mathrm{~min}$ after treatment had no protective effect. The implications of these findings for consolidation and retrieval models of RA were considered.
\end{abstract}

Several recent studies have demonstrated that inducing deep body cooling shortly after passive avoidance training results in a memory deficit $24 \mathrm{~h}$ later (e.g., Beitel \& Porter, 1968; Riccio, Hodges \& Randall, 1968; Riccio \& Stikes, 1969). This retrograde amnesia (RA) appears similar to the memory loss produced by ECS and other amnestic agents in that: (1) the magnitude of retention of loss is substantial, (2) there is a time-dependent relationship between the training-treatment delay and the severity of amnesia (Riccio et al., 1968), (3) electrical seizure-like activity occurs in the CNS during cooling (Vardaris, Gaebelein, \& Riccio, 1973), and (4) cortical temperature drop is produced by ECS (Oke, Mendelson, \& Justensen, 1974) as well as by hypothermic treatment (Lewis, Jackson, Miller, \& Misanin, 1972).

One problem with interpreting results from RA studies using hypothermia as the amnestic agent is that the depth and the duration of cooling are usually confounded. For instance, while Riccio et al. (1968) presented evidence that amnesia did not occur unless colonic temperature reached a point of $21.1^{\circ} \mathrm{C}$ or less, their procedure did not separate the magnitude of temperature loss from the total duration of the hypothermic state. Because recovery times were appreciably longer than the immersion

This investigation was supported, in part, by Grants GB-24220 and GB-41488 from the National Science Foundation. Portions of this paper were presented at the meeting of the Midwestern Psychological Association in Chicago, May 1973. Requests for reprints should be addressed to David Riccio, Department of Psychology, Kent State University, Kent, Ohio 44242. W. C. Wetsel is presently at the Department of Nutrition and Food Science, Massachusetts Institute of Technology, Cambridge, Massachusetts. period and varied as a function of the degree of original cooling, it is possible that total duration of the hypothermia condition, rather than absolute temperature loss, was the primary determinant of RA. More likely, perhaps, would be a tradeoff between duration and magnitude of hypothermia such that longer durations of moderate hypothermia will also produce RA.

An alternative position recently proposed by Misanin and Hoover (1971) is that the amnestic effects of hypothermia may depend primarily upon the extent to which new information or stimuli get processed after training. To examine this notion, they conducted an experiment in which recovery from hypothermia was modified by exposing rats to two different air temperatures for various periods of time following the immersion period. Animals maintained at lower temperatures for an extended period of time showed no memory loss, while those rewarmed relatively rapidly showed considerable RA. Misanin and Hoover (1971) interpreted this effect in terms of retrieval failure, and suggested that the rewarm treatment exposed these animals to greater stimulus inputs that probably "scrambled" retention processes and, therefore, produced the memory loss.

The present series of studies was designed to explore further the extent to which manipulating the duration of the hypothermic state modifies RA. In order to shorten the recovery period from deepbody cooling, subjects were artificially rewarmed by using warm water rather than air. Thus, hypothermia was induced and then reversed under several different experimental conditions. 


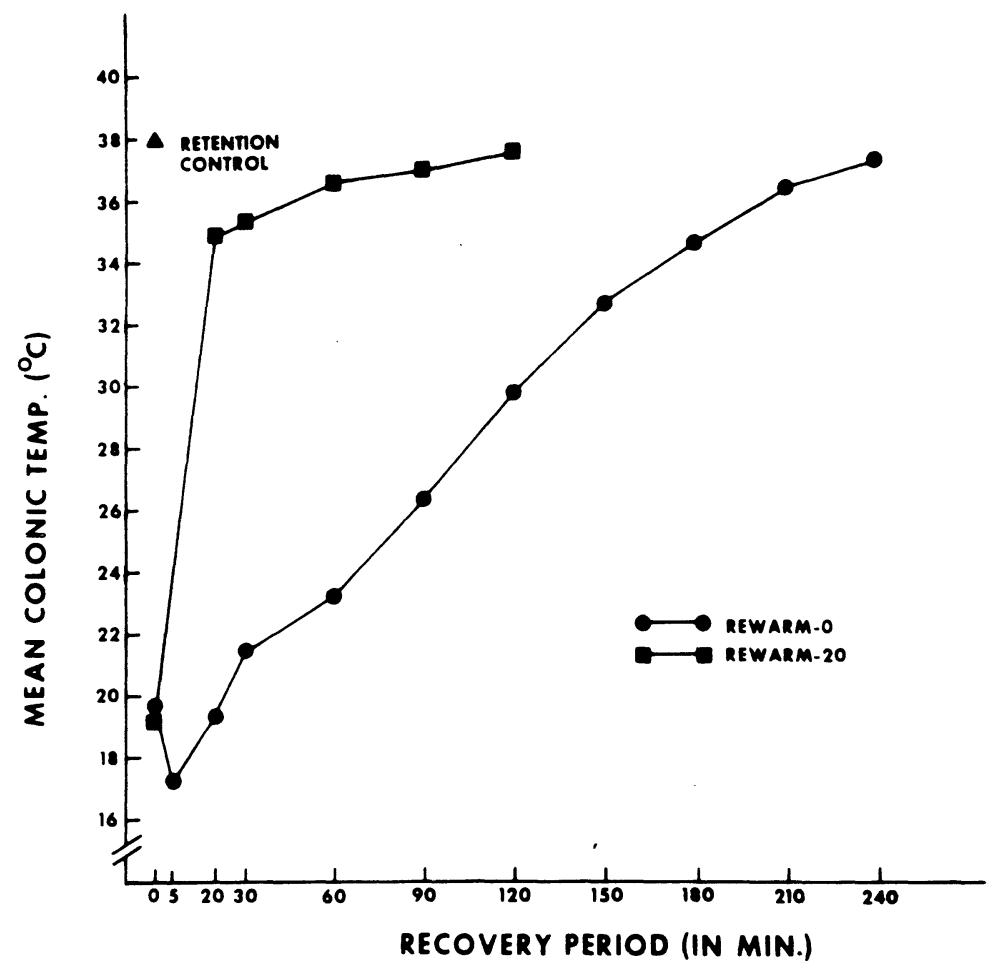

Figure 1. Mean colonic temperature during recovery period.

\section{EXPERIMENT I}

Experiment I examined the effect of slow and rapid recovery from hypothermia on a subsequent test of retention performance.

\section{Method}

Subjects. Thirty male, Holtzman, albino rats (357-414 g) served as the subjects in this first investigation. All animals were placed in individual cages 2 days prior to training. All rats had iree access to food and water in their home cages. Four rats assigned to the slow rewarm group (see procedure) died following immersion treatment and were replaced.

Apparatus. The major apparatus consisted of a $42.5 \times 21.2 \times$ $16.2 \mathrm{~cm}$ black-white passive avoidance chamber with a transparent Plexiglas lid. A partition with a $6.25 \times 8.75 \mathrm{~cm}$ guillotine door divided the box into two equal compartments. The white side contained a smooth aluminum plated floor, while the black compartment had a grid floor. These grids were connected to a crambled $170-\mathrm{V}$ shock from a matched impedance ac source described by Campbell and Teghtsoonian (1958).

All illumination was provided by a $15-\mathrm{W}$ bulb suspended above the center of the chamber. A mirror, mounted below the light source, permitted continuous observation of the subject by the experimenter. An electronic timer, calibrated to 10ths of a second, was used to monitor each animal's cross-through latencies. Wire cloth tubes were used to immerse the subjects in an ice water bath of $2^{\circ}-4^{\prime} \mathrm{C}$. Other apparatus consisted of a Model 2095 Forma-Temp. Jr. bath and circulator for the rewarm treatment and a Yellow Springs telethermometer with a probe for temperature measurements.

Procedure. On each of 2 days prior to training, all animals were individually handled for a 5 -min period. On the day of training, each subject was placed in the white compartment of the apparatus facing away from the door. Five seconds later a guillotine door was raised and a timer was started. Upon pla:ing all four feet into the black side of the apparatus, the guillotine door was closed and a 1 -sec inescapable shock was delivered to the rat. Following training, a retention control group $(n=10)$ was immediately placed in $36.4^{\circ} \mathrm{C}$ water for $20 \mathrm{~min}$, while experimentals were dunked in $2^{\circ}-4^{\circ} \mathrm{C}$ water until their colonic body temperatures reached approximately $19.3^{\circ} \mathrm{C}$. Of the two experimental hypothermia groups, a slow rewarm group $(n=10)$ was allowed to rewarm under room conditions of $21.1^{\circ}-23.8^{\circ} \mathrm{C}$, while a fast rewarm group $(n=10)$ was immersed in warm $\left(36.4^{\circ} \mathrm{C}\right)$ water for $20 \mathrm{~min}$. Subjects were then returned to their home cages until time of testing on the next day.

All subjects were tested for retention of the task $24 \mathrm{~h}$ later. The same procedure was employed as in training except that no shock was administered. Subjects failing to cross into the black chamber within 10 min uere assigned a test score of $600 \mathrm{sec}$. Again, cross-through scores were recorded.

\section{Results and Discussion}

Training latencies for the retention control, fast rewarm, and slow rewarm groups were 9.7, 6.4, and $11.4 \mathrm{sec}$, respectively. Due to very restricted variability, a small, but statistically significant, difference was found tetween the slow and fast rewarm groups $(U=16.5, \mathrm{p}<.02$, $\mathrm{ns}=10)$. No other differences were found among the training latencies. Because of the high proportion of ceiling scores obtained during testing, nonparametric two-tailed comparisons were made on all data throughout this paper except where otherwise noted.

Figure 1 presents the body temperature changes as a function of time after the hypothermic treatment. As is evident, the exposure to warm water for $20 \mathrm{~min}$ results in a rapid return to near normal body temperatures. Animals allowed to rewarm under room temperature conditions (our standard treatment) show a characteristic slight dip in temperature shortly after removal from cold water, then a gradual 
but steady increase in colonic temperature over several hours. The single point representing baseline temperature level is provided by the retention controls, which were never subjected to cooling.

The behavioral test data consist of cross-through latencies obtained $24 \mathrm{~h}$ after training. Median latency scores of 593.1, 582.5, and $125.9 \mathrm{sec}$ were obtained for retention controls, fast rewarm, and slow rewarm groups, respectively. A Kruskal-Wallis analysis indicated an overall main effect of treatment $(\mathrm{H}=$ $13.82, \mathrm{p}<.001, \mathrm{df}=2$ ). The slow rewarm group showed significantly shorter latencies than the retention controls $(U=2, p<.002, n s=10)$, indicating the typical hypothermia-induced amnesia. However, cross-through scores of the rapidly rewarmed group were significantly higher than those of the slow group $(U=6, p<.002$, ns $=10)$, and they failed to differ from the performance scores of retention controls. Thus, the results from the first experiment suggest that rate of rewarming following induction of hypothermia can markedly influence the extent of memory loss.

\section{EXPERIMENT II}

Because the colonic body temperature of the slow rewarm group continued to decrease by $2^{\circ} \mathrm{C}$ or more following removal from cold water, it was possible that the difference in memory loss between groups could be attributed to the absolute depth and not the rate of recovery from cooling. Although such an explanation seemed unlikely, Experiment II was designed to eliminate this confounding. A second purpose was to vary more systematically the rate of rewarming to further delineate its role in the prevention of retrograde amnesia.

\footnotetext{
Method

Subjects. Fifty naive male Holtzman albino rats, weighing between 348 and $479 \mathrm{~g}$, were used as the subjects in this second investigation. All animals were individually housed on the day of training and were given free access to food and water.

Apparatus. The apparatus for this second experiment was identical to that used in the first one.

Procedure. Passive avoidance training was identical to that of Experiment 1. Immediately following training, 40 experimentals were dunked in $2^{\circ}-4^{\circ} \mathrm{C}$ water, while 10 controls were returned to their home cages. The experimental animals consisted of a rewarm 0-min group cooled to $19.3^{\circ} \mathrm{C}$ and three other rewarm groups (5-, 7-, or 20 -min groups), which were cooled to $17.2^{\circ} \mathrm{C}$ in an effort to match the lowest temperature reached by the rewarm 0 -min group. After being cooled, subjects in the rewarm groups were immediately immersed in $36.4^{\circ} \mathrm{C}$ water for durations of $0,5,7$, or $20 \mathrm{~min}$ and then returned to their home cages. Six rats from the 0 -min group, 6 from the 5-min group, 5 from the 7 -min group, and 9 from the 20-min group died. These animals were replaced to keep 10 subjects in each group. All rats received retention testing $24 \mathrm{~h}$ later under the same procedure as that used in the first experiment.
}

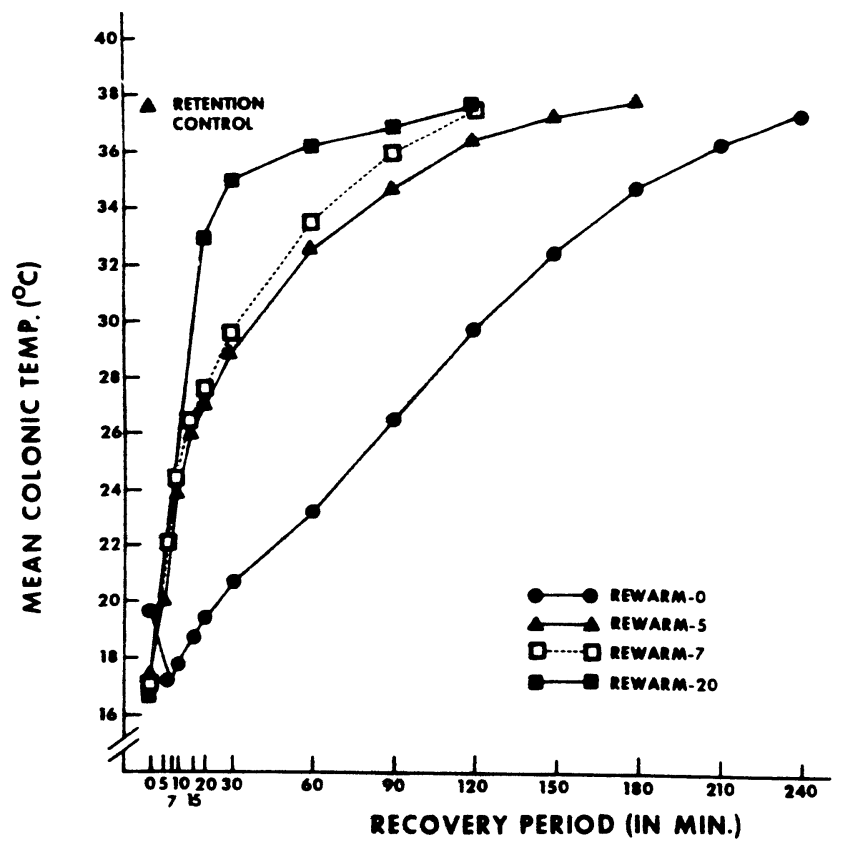

Figure 2. Mean colonic temperature during recovery session for groups given $0,5,7$, or 20 min immersion in warm water.

\section{Results and Discussion}

Median latencies obtained during training for the retention control, and the $0-, 5-, 7-$, and $20-\mathrm{min}$ rewarm groups were $6.7,6.6,9.5,7.1$, and $12.9 \mathrm{sec}$, respectively. No significant differences were found between any of the groups prior to testing.

The temperature recovery data for the five groups are displayed in Figure 2 . The additional postimmersion temperature dip still occurred in the nonrewarmed animals, but additional cooling for the other three groups was effective in producing approximately equivalent minimal body temperatures across conditions. As would be expected, there is a systematic relationship between duration of rewarming treatment and rate of body temperature recovery.

Figure 3 depicts the median cross-through latencies at testing as a function of the duration of rewarming treatment. A significant treatment effect $(\mathrm{H}=18.5, \mathrm{df}=4, \mathrm{p}<.001)$ was found when a Kruskal-Wallis analysis was applied to the data. The latencies of the three groups given brief or no rewarming treatments were significantly shorter than those of the retention controls (Us $\leqslant 8.5$, ps $<.002$, ns $=10$ ), suggesting the presence of retrograde amnesia. While there appears to be a curvilinear relationship with more memory loss at the intermediate rewarm intervals, this is not supported by statistical analysis $(\mathrm{H}=3.9$, $\mathrm{df}=2, \mathrm{p}>.1)$. Again, the 20-min rewarming period was effective in preventing RA, as these subjects did not differ from retention con- 


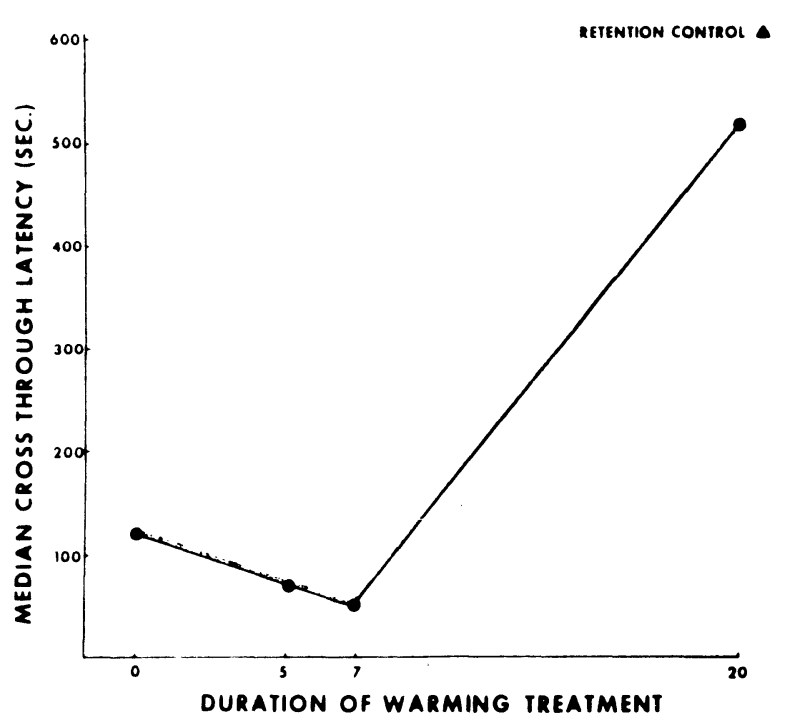

Figure 3. Median passive avoidance test latencies obtained for rats given hypothermia treatment followed immediately by immersion in warm water for a duration of $0,5,7$, or $20 \mathrm{~min}$.

trois $(\mathrm{U}=32, \mathrm{p}>.1, \mathrm{n}=10)$ but did differ from each of the other rewarming conditions (Us $\leqslant 11$, ps $<.02$, ns $=10$ ). This result replicates the findings of Experiment $I$ and further indicates that the previous outcome was not an artifact of the slightly greater temperature drop in the slow-recovery group.

\section{EXPERIMENT III}

The preceding experiments demonstrate that the usual RA produced by deep body cooling is prevented when subjects are rapidly rewarmed following the hypothermia treatment. This reversability of effects suggests another way in which to examine the temporally graded nature of RA. Ordinarily, time-dependent changes are assessed by delaying the interval between training and the amnestic treatment. In the present case, the amnestic treatment was given immediately for all groups, but the time until onset of rewarming was varied. If the duration of hypothermia is an important determinant of RA, then delaying the rewarming treatment should result in increasing the magnitude of RA.

\section{Method}

Subjects. Forty adult males, similar to those described in the second experiment, served as subjects in Experiment III.

Apparatus. All apparatus used in this experiment were the same as those employed in the previous experiments.

Procedure. Five groups of eight subjects were given passive avoidance training followed immediately by hypothermia treatment, as described in Experiment II. Four groups of subjects were rewarmed by immersion in $36.4^{\circ} \mathrm{C}$ water for $20 \mathrm{~min}$, either $0,5,10$, or $30 \mathrm{~min}$ after completion of the hypothermia treatment. A fifth group, the amnesia control, received no rewarming treatment. A total of 2, 5, 2, 4, and 6 rats assigned to the amnesia control and the $0-, 5-, 10-$, and 30-min groups, respectively, died following immersion treatment and were replaced. All subjects were tested $24 \mathrm{~h}$ after training.

\section{Results}

As in Experiment II, no significant differences were found between any of the groups during training. The median cross-through latencies for the 0 -, 5-, 10-, 30-min delay rewarm groups and the amnesia control groups were 8.6, 24.7, 18.2, 12.9, and $8.6 \mathrm{sec}$, respectively.

Median cross-through latencies obtained during testing are presented in Figure 4. A Kruskal-Wallis analysis of variance performed on these data indicated a significant treatment effect $(\mathrm{H}=13.28, \mathrm{df}=4$, $\mathrm{p}<.01)$. No differences were obtained between the three groups rewarmed 0,5 , or $10 \mathrm{~min}$ after hypothermia. However, the group rewarmed $30 \mathrm{~min}$ after hypothermia had significantly shorter latencies than both the groups rewarmed 0 to $10 \mathrm{~min}$ after hypothermia (Us $\leqslant 11$, ps $<.03$, ns $=8$ ) and the group rewarmed $5 \mathrm{~min}$ after hypothermia treatment
Figure 4. Median cross-through latencies of groups tested $24 \mathrm{~h}$ after passive avoidance training. All rats received hypothermia treatment immediately after training and then either no artificial rewarming (amnesia control) or rewarming after a delay of 0 , 5,10 , or $30 \mathrm{~min}$.

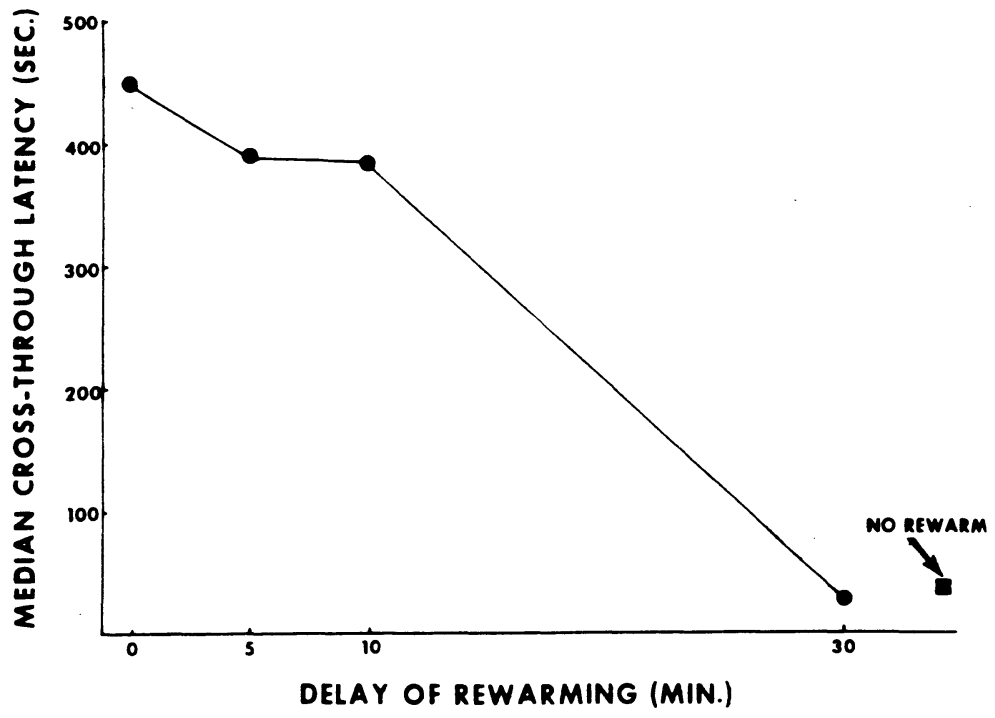


( $U=15, p<.04, n=8$, one-tailed test). These results indicate that delaying the rewarm treatment reduces its effectiveness in preventing $R A$.

Further evidence that the rewarming must occur relatively quickly following the hypothermia treatment was indicated by individual comparisons between the amnesia control group and the four other rewarm groups. The 0-, 5-, and 10-min delay groups all had significantly higher retention crossthrough latencies than the no-rewarm group (Us $<16.5$, $.003<\mathrm{ps}<.06$, ns $=8$, one-tailed test). No significant difference was found, however, between the groups receiving no rewarming or rewarming after a 30-min delay $(U=28, p>.3$, one-tailed test $)$. In general, the results of this experiment indicate that the total duration of hypothermia (i.e., time to reach normothermic levels) plays an important role in producing amnesia. If the rewarming treatment utilized in this experiment was initiated within $10 \mathrm{~min}$ after the hypothermia treatment, little evidence for RA was obtained. But, if initiation of the treatment to restore body temperatures to near normal levels was delayed for $30 \mathrm{~min}$, marked retention loss occurred.

\section{DISCUSSION}

The first two experiments demonstrate that RA produced by deep body cooling in rats can be attenuated by rapid rewarming. While this conclusion appears to contrast with that drawn by Misanin and Hoover (1971), the discrepancy may reflect primarily the type of rewarming manipulation employed. Indeed, the substantial RA seen in Experiment II in our briefly rewarmed groups ( 5 or $7 \mathrm{~min}$ ) seems similar to the findings reported by Misanin and Hoover (1971) in one of their rewarming conditions. Our use of warm water as the recovery treatment may have permitted us to include a broader range of recovery rates than was possible in their study.

While the first two experiments show that there is virtually no disruption of memory if hypothermia is followed by rapid rewarming for $20 \mathrm{~min}$, Experimeni III demonstrates that, to be effective, this manipulation must be instituted shortly after cooling. As the delay between hypothermia and recovery treatment increases, the magnitude of memory loss also increases. Thus, with an interval of only $30 \mathrm{~min}$ between completion of cooling and onset of rewarming, the magnitude of RA is entirely comparable to that of amnestic controls which were not rewarmed. In contrast, initiation of rewarming within 10 min completely prevented RA.

At a theoretical level, one interpretation of the present results is that the memory engram is not impaired unless the disruption of CNS events associated with hypothermia is allowed to persist in time. Although major changes in temperature (Lewis et al.,
1972) and electrical activity (e.g., Vardaris et al., 1973 ) in the CNS have presumably occurred during and shortly after hypothermia induction, it could be argued that rapid and early rewarming prevents RA by reinstating normal physiological functioning. Whether recovery of metabolic and electrical processes of the CNS closely parallel the rewarming conditions remains a moot question for now.

An alternative interpretation (which we currently favor) is based upon the notion that retrieval deficits, rather than storage failures, are central to hypothermia-induced RA. While this study shows that memory loss is eliminated by reversing the treatment, other findings show that RA is attenuated also by restoring treatment conditions at the test session. More specifically, we have recently reported that subjects tested within several hours after hypothermic treatment, or reexposed to cooling prior to testing at longer intervals, show little evidence of the typical RA (Hinderliter, Webster, \& Riccio, 1975). Thus, the presence or absence of memory appears to be controlled by specific stimulus conditions. Our retrievaloriented interpretation, similar but not identical to state dependent views of $\mathrm{RA}^{-}$(e.g., Thompson \& Grossman, 1972), assumes that processing continues after training and that the memory becomes linked with contextual cues related to the hypothermic condition. Memory is then retrievable only when the encoding stimuli are present, as in testing at short intervals or following a recooling treatment. Applying this analysis to the present study, rapid rewarming shortly after acquisition may simply provide an opportunity for memory processes to become encoded with respect to normal as well as abnormal body states. During testing in the normothermic condition, sufficient retrieval cues presumably are available to provide access to memory. Sufficient delay of rewarming would increase the liklihood that memory is uniquely encoded with respect to hypothermia (and related) cues, and, as in Experiment III, would produce marked memory loss.

Finally, it is of interest that one consequence of ECS treatment is a period of cortical hypothermia (Oke et al., 1974). Oke et al. have suggested that these changes in brain temperature may play a crucial role in ECS-induced RA, and proposed that treatments which prevent temperature loss might also preserve memory. Our evidence that rapid rewarming following hypothermia treatment prevents RA seems congruent with the general tenor of their hypothesis.

\section{REFERENCES}

Beitel, R. E., \& Porter, P. B. Deficits in short- and long-term retention and impairments in learning induced by severe hypothermia in mice. Journal of Comparative and Physiological Psychology, 1968, 66, 53-59. 
Campbell, B. A., \& Teghtsoonian, R. Electrical and behavioral effects of different types of shock stimuli on the rat. Journal of Comparative and Physiological Psychology, 1958, 51, 185-192.

Hinderliter, C. F., Webster, T., \& Riccio, D. C. Amnesia induced by hypothermia as a function of treatment-test interval and recooling in rats. Animal Learning \& Behavior, 1975, 3. $257-263$.

Lewis, D. J., Jackson, F., Miller, R. R., \& Misanin, J. R. The relation between rectal and brain temperatures following hypothermia. Psychonomic Science, 1972, 27, 29-30.

Misanin, J. R., \& Hoover, M. Recovery rate as a determinant of the amnesia-like effect of hypothermia. Physiology and Behavior, 1971, 6, 689-693.

Oke, A. F., Mendelson, J., \& Justensen, D. R. Cortical hypothermia is a sequela of electroconvulsive shock. Nature, 1974, 248, 437-439.

Riccio. D. C., Hodges, L. A., \& Randall, P. K. Retrograde amnesia produced by hypothermia in rats. Journal of Comparative and Physiological Psychology, 1968, 66, 618-622.

Riccio, D. C., \& Stukes, E. R. Persistent but modifiable retrograde amnesia produced by hypothermia. Physiology and Behavior, $1969,4,649-652$.
ThOMPSON, C. I., \& Grossman, L. B. Loss and recovery of long-term memories after ECS in rats: Evidence for statedependent recall. Journal of Comparative and Physiological Psychology, 1972, 78, 248-254.

Vardaris, R. M., Gaebelein, C., \& Riccio, D. C. Retrograde amnesia from hypothermia-induced brain seizures. Physiological Psychology, 1973, 1, 204-208.

\section{NOTE}

1. Although a large proportion of rats died in Experiments II and III, there does not appear to be any systematic relationship between behavioral outcomes and mortality rate. For example, rats warmed for $20 \mathrm{~min}$ immediately after training-hypothermia treatment in both Experiments II and III behaved similarly during retention testing but differed markedly in the proportion of fatalities.
(Received for publication September 25, 1975; revision received December 24,1975 .) 